\title{
Estudo CUMPRO: CUMPrimento do Rastreio Oncológico em profissionais de um ACeS
}

Margarida Moreira, ${ }^{1}$ Aníbal Martins, ${ }^{2}$ Vera Amaral, ${ }^{2}$ Rute Mota, ${ }^{2}$ Ana Almeida, ${ }^{3}$ Carla Morna ${ }^{4}$

\section{RESUMO}

Objetivo: As doenças oncológicas são a segunda principal causa de morte em Portugal. O Plano de Vigilância Oncológica Nacional contempla os rastreios do cancro colorretal (CCR), da mama e do colo do útero (CCU). O objetivo primário deste trabalho foi determinar a proporção de profissionais de um Agrupamento de Centros de Saúde (ACeS) que os cumpre efetivamente. Tipo de estudo: Estudo censitário, observacional transversal descritivo.

População: Profissionais do ACeS Póvoa de Varzim/Vila do Conde elegíveis para os rastreios oncológicos referidos.

Métodos: Aplicou-se um questionário anónimo sobre a adesão ao rastreio oncológico e motivos de não adesão se aplicável. A distribuição dos questionários foi efetuada entre 9 de fevereiro e 4 de abril de 2015.

Resultados: A taxa de resposta foi de 87,0\%. Foram obtidos 267 questionários de um total de 307 participantes elegíveis. Oito questionários foram excluídos. A mediana das idades situou-se nos 41 anos. Na globalidade, 82,2\% dos profissionais apresentavam rastreios atualizados de acordo com a sua idade e género. Numa base individual, $77,8 \%$ dos elegíveis apresentava o rastreio do CCR atualizado, $84,1 \%$ o do cancro da mama e $87,6 \%$ o do CCU.

Conclusões: Verificou-se uma boa adesão aos rastreios oncológicos pelos profissionais. Este estudo poderá servir como termo comparativo para futuras investigações.

Palavras-chave: Deteção Precoce do Cancro; Profissionais de Saúde; Adesão a Recomendações.

\section{INTRODUÇÃO}

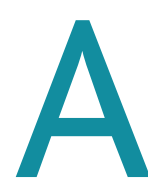

s doenças oncológicas constituem a segunda principal causa de morte em Portugal (apenas precedidas pelas doenças do aparelho circulatório) - 25,0\% das mortes em 2014, segundo o PORDATA ${ }^{1}$ - e têm um profundo impacto nos doentes, nos familiares e na sociedade, sendo provavelmente das doenças mais temidas pela população em geral. ${ }^{2}$ Muitas das mortes por cancro seriam evitáveis através de medidas de prevenção primária e secundária (diagnóstico precoce em rastreios), ${ }^{3}$ pelo que o reconhecimento destes factos levou a estabelecer o

${ }^{1}$ Médica Interna de Medicina Geral e Familiar. USF das Ondas ${ }^{2}$ Médico Interno de Medicina Geral e Familiar. USF Santa Clara ${ }^{3}$ Médica Especialista de Medicina Geral e Familiar. USF Santa Clara ${ }^{4}$ Médica Especialista de Medicina Geral e Familiar. USF das Ondas combate contra o cancro como uma das prioridades do Plano Nacional de Saúde. ${ }^{2}$

No entanto, as recomendações para rastreio variam consoante as sociedades científicas. Em Portugal, o Plano de Vigilância Oncológica Nacional vigora desde 1990, tendo como objetivo a deteção precoce da doença oncológica, visando a redução da mortalidade por esta causa ${ }^{4}$ de acordo com a relação custo-efetividade dos rastreios. Assim, o Plano Nacional de Prevenção e Controlo das Doenças Oncológicas (PNPCDO) atualizado em 2012 recomenda, em pessoas sem outros fatores de risco para malignidade:

- Rastreio do cancro do colo útero (CCU) através de citologia cérvico-vaginal nas mulheres com idade entre os 25-30 e os 60 anos;

- Rastreio do cancro da mama por mamografia a cada dois anos nas mulheres dos 50 aos 69 anos; 
- Rastreio do cancro colorretal (CCR) através da pesquisa de sangue oculto nas fezes (PSOF) em homens e mulheres dos 50 aos 74 anos. $^{2-3}$

Adicionalmente, em relação ao rastreio oportunístico do CCR, encontra-se em discussão pública uma norma da Direção-Geral da Saúde de 2014, ${ }^{5}$ que advoga o uso da PSOF anualmente e estabelece as situações em que a colonoscopia deve ser utilizada.

De encontro ao PNPCDO e no contexto dos processos de contratualização e monitorização dos Cuidados de Saúde Primários (CSP), à luz da evidência científica, normas e orientações atualmente existentes, os bilhetes de identidade dos indicadores ${ }^{6}$ utilizados neste âmbito, em 2014, tinham em consideração:

1. A proporção de mulheres entre [25; 60] anos com, pelo menos, uma colpocitologia nos últimos três anos;

2. A proporção de mulheres entre $[50 ; 70]$ anos com, pelo menos, uma mamografia registada nos últimos dois anos;

3. A proporção de utentes com idade entre $[50 ; 75]$ anos com rastreio de cancro do cólon e reto efetuado, contando para isso: pelo menos um resultado de PSOF nos últimos dois anos ou, pelo menos, um resultado de retossigmoidoscopia nos últimos cinco anos ou um resultado de colonoscopia nos últimos dez anos. Não obstante os critérios utilizados, em Portugal, à semelhança do que acontece em outros países da União Europeia, a magnitude da execução do rastreio oncológico fica aquém do desejado. ${ }^{2}$ Entre os fatores que explicam as discrepâncias entre as recomendações existentes e a prática, encontram-se aqueles associados ao médico, incluindo a falta inconsciente de motivação própria para a recomendação em causa. ${ }^{7-8}$

No caso particular dos médicos de família, um estudo realizado em Israel demonstrou que $66,7 \%$ dos participantes referia não participar em qualquer atividade preventiva, apesar da importância que lhes conferia na melhoria da qualidade dos cuidados prestados, sobretudo quando instituídas como uma prática corrente. ${ }^{9}$

Neste contexto, alguns estudos internacionais versaram sobre o rastreio oncológico em profissionais de saúde e chegaram a resultados diversos. Em Portugal, os estudos sobre este tema são muito escassos, especialmente os que envolvem os vários rastreios oncológicos.
Assim, os objetivos deste trabalho foram: determinar a proporção de profissionais do Agrupamento de Centros de Saúde (ACeS) Póvoa de Varzim/Vila do Conde (PV/VC) com idade para realização de rastreio oncológico (CCR, cancro da mama e CCU), que o cumpre efetivamente, de acordo com os bilhetes de identidade dos indicadores mencionados; relacionar esses resultados com algumas características destes profissionais; e estudar os motivos de não cumprimento, quando aplicável.

\section{MÉTODOS}

Realizou-se um estudo censitário, observacional, transversal e descritivo, recorrendo a um questionário original (Anexo I), elaborado pelos autores, que foi previamente submetido a um estudo-piloto numa amostra de conveniência de um outro ACeS da Região Norte para aperfeiçoamento e retificação do documento.

A população em estudo incluiu todos os funcionários em funções no ACeS PV/VC, a ele pertencentes ou mobilizados para o mesmo com idade para rastreio oncológico. A listagem total dos profissionais foi disponibilizada pela Unidade de Apoio à Gestão (UAG) em janeiro de 2015, nela figurando 361 elementos.

Como critérios de exclusão consideraram-se: funcionários do sexo masculino com menos de 50 anos de idade (à data da recolha de dados); funcionários do sexo feminino com menos de 25 anos (à data da recolha de dados); funcionários do ACeS a exercer funções noutro local; funcionários do Instituto do Emprego e Formação Profissional; e prestadores de serviços de empresas contratadas (segurança, limpeza). Adicionalmente, questionários com dados sociodemográficos preenchidos de forma incompleta e/ou inadequada não foram considerados na análise.

Por motivos de simplificação logística foi determinado, antes de iniciar a colheita de dados, distribuir o questionário a todos os profissionais $(n=361)$. Deste modo, os questionários correspondentes a homens com menos de 50 anos ou mulheres com menos de 25 seriam posteriormente rejeitados do tratamento de dados por apresentarem critérios de exclusão.

Em janeiro de 2015, os autores contactaram os coordenadores de todas as Unidades do ACeS (contactos fornecidos pela UAG do ACeS), no sentido de solicitar autorização para a apresentação do estudo em questão 
(texto padronizado e demonstração do questionário por correio eletrónico) e distribuição dos questionários, preferencialmente em reunião de serviço em data a acordar (ou, se tal não fosse possível, noutro dia a combinar, conforme a disponibilidade).

No contacto com os profissionais de saúde foi fornecido um questionário de autopreenchimento, confidencial e anónimo para preenchimento imediato ou até um prazo máximo de uma semana, que deveria depois ser colocado em urna própria, deixada para o efeito, a ser levantada pelos investigadores. Os dados foram recolhidos entre 9 de fevereiro e 4 de abril de 2015.

As variáveis estudadas foram: idade, género, estado civil, habilitações académicas, função exercida, local onde exerce (para aqueles alocados a mais que uma Unidade considerou-se aquela a que dedica mais tempo), hábitos tabágicos, hábitos etílicos, atividade física, ter médico de família, ter tido uma consulta presencial com o médico de família nos últimos três anos, estar inscrito em Unidade de Saúde Familiar (USF) do ACeS PV/VC, ter tido pelo menos uma avaliação da tensão arterial nos últimos dois anos, ter recebido vacina antigripal no ano anterior, ter vacinação antitetânica atualizada, cumprimento do rastreio do CCR, CCU e cancro da mama de acordo com o preconizado pelos indicadores contratualizados nos CSP em $2014^{6}$ e motivos para o não cumprimento dos mesmos nos casos em que tal se verificava. Neste trabalho optou-se por utilizar os critérios de rastreio definidos pelos indicadores, de modo a possibilitar a comparação com os resultados obtidos no acompanhamento dos utentes do mesmo ACeS.

Relativamente aos hábitos etílicos, considerou-se consumo de baixo risco aquele não superior a sete unidades-padrão por semana na mulher ou não superior a catorze unidades por semana no homem, ou consumo excessivo aquele que ultrapassasse os números referidos. ${ }^{10} \mathrm{~A}$ atividade física foi considerada se presente, em média, durante pelo menos 30 minutos no mínimo cinco dias por semana. ${ }^{11}$

Os investigadores contaram com o consentimento presumido dos intervenientes no estudo. O presente estudo teve parecer favorável da Comissão de Ética para a Saúde da Administração Regional de Saúde do Norte.

Os dados recolhidos foram organizados numa base de dados utilizando o programa Microsoft Office Excel
$2011 @$, tendo posteriormente sido validados e analisados através do programa IBM® SPSS ${ }^{\circledR}$ Statistics 19.

Os dados foram sumarizados utilizando estatísticas descritivas, nomeadamente média e desvio-padrão/mediana, mínimo e máximo para variáveis numéricas e frequências absolutas e relativas para variáveis categóricas. A análise descritiva realizada pelos autores deste estudo foi integralmente revista e validada através do Clinical Lab - Serviço de Consultoria para MGF, disponibilizado por Eurotrials ${ }^{\circledR}$ em parceria com Merck Sharp \& Dohme ${ }^{\circledR}$ e com patrocínio científico da Ordem dos Médicos. Não foi realizada análise estatística inferencial por se tratar de um estudo censitário, em que se pretendeu estudar a totalidade da populaçãoalvo.

\section{RESULTADOS}

Foram obtidos 313 questionários respondidos. Destes, 46 foram excluídos por corresponderem a homens com menos de 50 anos.

Atendendo a que do total de funcionários do ACeS faziam parte 54 homens sem idade para rastreio, obteve-se uma taxa de resposta do público-alvo de $87,0 \%$ (267/307).

Posteriormente, foram ainda excluídos oito participantes do tratamento de dados por não terem preenchido corretamente os dados sociodemográficos, conforme exigido pelos critérios de seleção.

\section{Caracterização da população}

O Quadro I mostra a caracterização geral dos participantes. Nenhum profissional assinalou exercer numa Unidade de Cuidados de Saúde Personalizados, uma vez que no ACeS em questão operam apenas USFs.

A idade dos profissionais incluídos variou entre $25 \mathrm{e}$ 64 anos, com uma média de 42,4 anos e mediana de 41 anos (Figura 1). Verificou-se um predomínio de participantes do sexo feminino, casados ou a viver em união de facto e com habilitações literárias ao nível do ensino superior. No contexto profissional, aproximadamente um terço dos participantes eram enfermeiros e a maioria $(81,5 \%)$ exercia em USFs. Relativamente aos estilos de vida, a maioria dos participantes referiu nunca ter fumado $(69,9 \%)$ e ter hábitos etílicos de baixo risco $(99,6 \%)$. Menos de metade dos participantes referiu praticar atividade física. 
A maioria dos participantes referiu estar inscrita em lista de médico de família (97,3\%), tendo comparecido a pelo menos uma consulta com o seu médico nos últimos três anos $(86,1 \%)$. Registou-se ainda um predomínio dos profissionais inscritos numa USF deste ACeS (75,7\%). Relativamente a outras medidas preventivas, a maioria tinha medido a tensão arterial nos últimos dois anos e tinha a vacinação antitetânica atualizada $(98,1 \%)$. Pouco mais de metade dos participantes assinalou ter recebido a vacina da gripe no ano anterior.

\section{Cumprimento global do rastreio oncológico}

Relativamente ao cumprimento global de rastreio oncológico, $82,2 \%$ dos participantes apresentavam rastreios atualizados de acordo com a sua idade e género.

\section{Cumprimento do rastreio do CCR}

Dos 81 participantes com idade para rastreio do CCR, 77,8\% ( $n=63$ ) mencionaram tê-lo feito de acordo com os critérios estabelecidos. A proporção de cumprimento do rastreio do CCR foi de $78,3 \%$ nas mulheres e de $75,0 \%$ nos homens.

Quanto ao método de rastreio, 34,9\% ( $n=22)$ dos participantes, dois homens e 20 mulheres, foram rastreados através de pesquisa de sangue oculto nas fezes, $44,4 \%$ ( $n=28)$, cinco homens e 23 mulheres, através de colonoscopia total e 20,6\% ( $n=13$ ), dois homens e 11 mulheres, através de ambos os métodos.

Nas mulheres, o motivo mais frequentemente assinalado (Quadro II) para o não cumprimento de rastreio do CCR foi «O seu médico não lhe recomendou», enquanto nos homens foi «Falta de tempo» (Quadro II).

\section{Cumprimento do rastreio do $\mathrm{CCU}$}

Das 234 mulheres em idade para rastreio do CCU, $87,6 \%$ ( $n=205$ ) referiram tê-lo atualizado. As razões assinaladas pelas mulheres para o não cumprimento do rastreio foram

\begin{tabular}{|c|c|}
\hline \multicolumn{2}{|l|}{ Características } \\
\hline Idade em anos, mediana (mínimo; máximo) & 41 anos $(25 ; 64)$ \\
\hline \multicolumn{2}{|l|}{ Género, $n(\%)$} \\
\hline Masculino & $12(4,6 \%)$ \\
\hline Feminino & $247(95,4 \%)$ \\
\hline \multicolumn{2}{|l|}{ Estado civil, $n(\%)$} \\
\hline Solteiro/a & $59(22,8 \%)$ \\
\hline Casado/a ou em união de facto & $166(64,1 \%)$ \\
\hline Separado/a ou divorciado/a & $33(12,7 \%)$ \\
\hline Viúvo/a & $1(0,4 \%)$ \\
\hline \multicolumn{2}{|l|}{ Habilitações académicas, $n$ (\%) } \\
\hline $1 .^{\circ}-4 .^{\circ}$ ano do ensino básico & $2(0,8 \%)$ \\
\hline $5 .^{\circ}-9 .^{\circ}$ ano do ensino básico & $17(6,6 \%)$ \\
\hline $10 .^{\circ}-12 .^{\circ}$ ano do ensino secundário & $41(15,8 \%)$ \\
\hline Bacharelato/Licenciatura/Mestrado/Doutoramento & $198(76,4 \%)$ \\
\hline Outras & $1(0,4 \%)$ \\
\hline \multicolumn{2}{|l|}{ Função exercida, $n$ (\%) } \\
\hline Enfermeiro/a & $85(32,8 \%)$ \\
\hline Médico/a especialista & $63(24,3 \%)$ \\
\hline Médico/a interno/a & $36(13,9 \%)$ \\
\hline Técnico superior & $9(3,5 \%)$ \\
\hline Assistente operacional & $14(5,4 \%)$ \\
\hline Assistente técnico & $50(19,3 \%)$ \\
\hline Outra & $2(0,8 \%)$ \\
\hline \multicolumn{2}{|l|}{ Local onde exerce, $n$ (\%) } \\
\hline USF & $211(81,5 \%)$ \\
\hline URAP & $7(2,7 \%)$ \\
\hline UCC & $15(5,8 \%)$ \\
\hline USP & $10(3,9 \%)$ \\
\hline UAG & $14(5,4 \%)$ \\
\hline Outros & $2(0,8 \%)$ \\
\hline \multicolumn{2}{|l|}{ Hábitos tabágicos, $n$ (\%) } \\
\hline Fumador & $44(17,0 \%)$ \\
\hline Ex-fumador & $34(13,1 \%)$ \\
\hline Nunca fumou & $181(69,9 \%)$ \\
\hline \multicolumn{2}{|l|}{ Hábitos etílicos, $n$ (\%) } \\
\hline Consumo de baixo risco & $258(99,6 \%)$ \\
\hline Consumo excessivo & $1(0,4 \%)$ \\
\hline \multicolumn{2}{|l|}{ Atividade física, $n$ (\%) } \\
\hline Sim & $111(42,9 \%)$ \\
\hline Não & $148(57,1 \%)$ \\
\hline \multicolumn{2}{|l|}{ Tem médico de família, $n$ (\%) } \\
\hline Sim & $252(97,3 \%)$ \\
\hline Não & $7(2,7 \%)$ \\
\hline
\end{tabular}




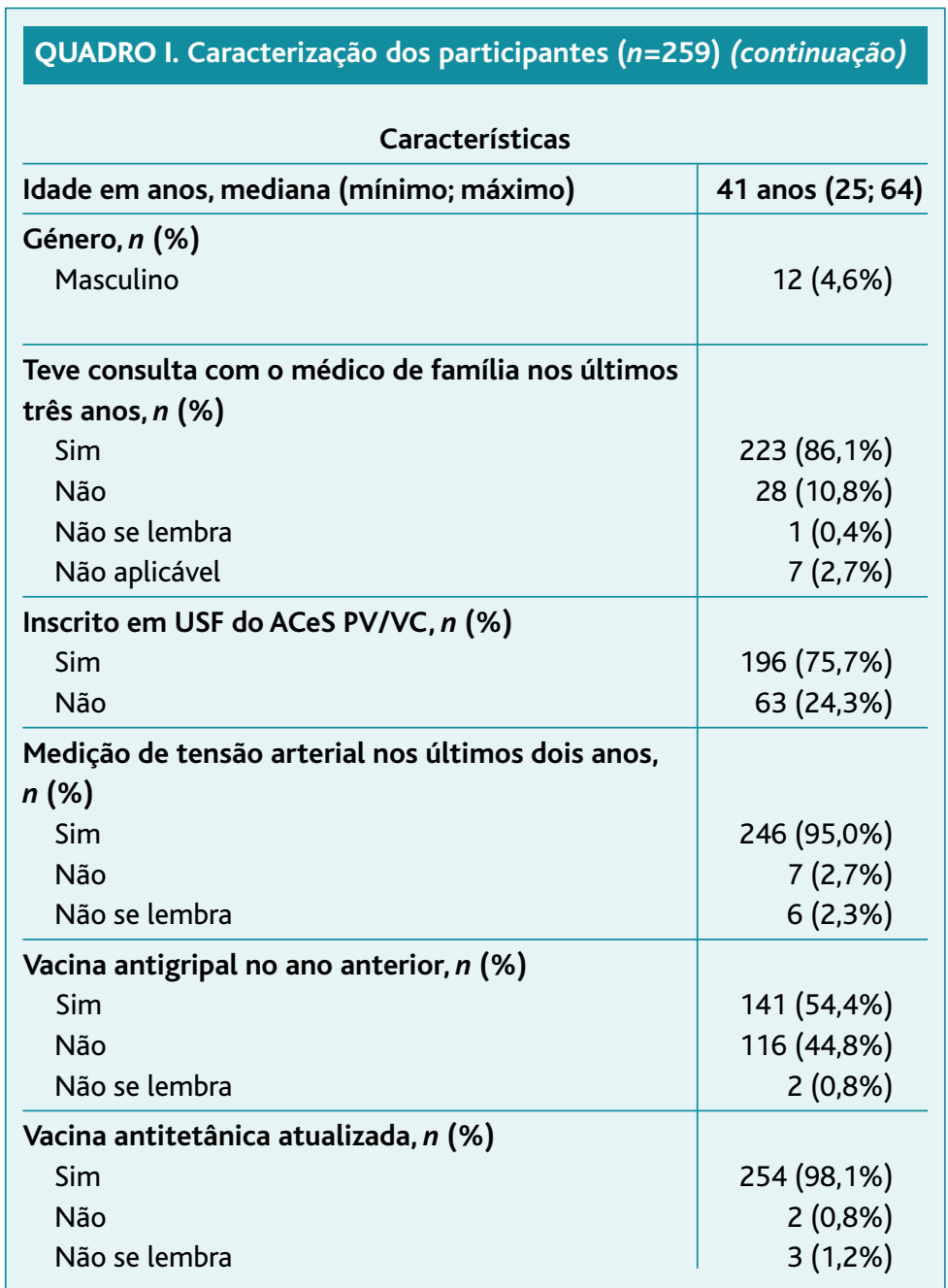

Legenda: ACeS PV/VC - Agrupamento de Centros de Saúde Póvoa de Varzim / Vila do Conde; UAG - Unidade de Apoio à Gestão; UCSP - Unidade de Cuidados de Saúde Personalizados, UCC - Unidade de Cuidados na Comunidade; URAP - Unidade de Recursos Assistenciais Partilhados; USF - Unidade de Saúde Familiar; USP - Unidade de Saúde Pública.

«Não quis fazer» ( $n=7)$, «Falta de tempo» $(n=7)$, «Esquecimento» ( $n=5)$, «Sem indicação» $(n=3)$, «Thinprep há quatro anos» $(n=1)$, «O seu médico não recomendou» $(n=1)$ e «Outra razão» $(n=3)$. Duas mulheres não justificaram o motivo de não cumprimento do rastreio.

\section{Cumprimento do rastreio do cancro da mama}

Das 69 mulheres participantes com critérios para realização do rastreio do cancro da mama, $84,1 \%(n=58)$ responderam ter rastreio atualizado. Entre os motivos mencionados para não o ter, encontram-se «Não quis fazer» (n=7), «Não acha importante» $(n=1) \mathrm{e}$ «Outra razão» ( $n=2)$. Uma mulher não justificou o facto de não ter o rastreio atualizado.

\section{Cumprimento do rastreio oncológico e características dos participantes}

Relativamente à idade mediana, verifica-se que os profissionais que cumpriram o rastreio do CCR são quatro anos mais velhos do que os que não cumpriram; as mulheres que fizeram rastreio do CCU são oito anos mais novas do que as que não o fizeram, enquanto no cancro da mama a idade mediana das mulheres que cumpriram foi exatamente igual à das mulheres que não cumpriram.

O Quadro III apresenta a proporção de cumprimento com cada um dos rastreios, de acordo com as características do grupo de profissionais.

Destacam-se, de seguida, as diferenças encontradas nas proporções de cumprimento dos rastreios entre profissionais com diferentes características, assumindo como critério empírico uma diferença superior a $10 \%$ (e não sendo referidas quando a maioria dos profissionais se concentrava numa única classe). No rastreio do CCR, os profissionais que frequentaram o ensino superior apresentavam uma adesão mais elevada do que aqueles com nível de escolaridade inferior $(83,0 \% v s$. $70,4 \%$ ), assim como os não fumadores comparativamente aos fumadores $(80,6 \% v s$. $64,3 \%)$, os divorciados/viúvos comparativamente aos solteiros $(84,2 \%$ vs. $70,0 \%)$, os médicos comparativamente aos outros profissionais $(82,9 \%$ vs. $73,9 \%)$ e os profissionais que exercem em USFs comparativamente aos que exercem nas restantes tipologias $(80,6 \%$ vs. $68,4 \%)$.

No rastreio do CCU, o cumprimento foi superior nos enfermeiros comparativamente aos médicos $(92,7 \% v s$. $81,9 \%)$.

No rastreio do cancro da mama, o cumprimento foi superior nos não fumadores comparativamente aos fumadores $(87,7 \%$ vs. $66,7 \%)$ e nos profissionais de unidades não USF comparativamente aos profissionais em USFs $(93,8 \%$ vs. 81,1\%). 


\section{QUADRO II. Motivos do não cumprimento do rastreio do cancro CCR $(n=16)$}

\begin{tabular}{l|c|c|c} 
Motivo & Mulheres $-\boldsymbol{n}(\%)$ & Homens $-\boldsymbol{n}(\%)$ & Total $-\boldsymbol{n}(\%)$ \\
\hline Não quis fazer & $2(15,4 \%)$ & $0(0,0 \%)$ & $2(12,5 \%)$ \\
\hline O seu médico não lhe recomendou & $7(53,8 \%)$ & $0(0,0 \%)$ & $7(43,8 \%)$ \\
\hline Falta de tempo & $2(15,4 \%)$ & $2(66,7 \%)$ & $4(25,0 \%)$ \\
\hline Esquecimento & $0(0,0 \%)$ & $0(0,0 \%)$ & $0(0,0 \%)$ \\
\hline Não acha importante & $0(0,0 \%)$ & $0(0,0 \%)$ & $0(0,0 \%)$ \\
\hline Outra razão & $2(15,4 \%)$ & $1(33,3 \%)$ & $3(18,8 \%)$
\end{tabular}

e género $(82,2 \%)$. Relativamente ao total de 53 motivos evocados para o não cumprimento dos rastreios, destacase o «Não quis fazer» como o mais frequente $(n=16)$, seguido pela «Falta de tempo» ( $n=11)$, «Outra razão» $(n=10)$, «O seu médico não lhe recomendou» ( $n=8)$, «Esquecimento» ( $n=5)$ e «Não acha importante» $(n=1)$.

\section{DISCUSSÃO}

Neste estudo verificou-se uma elevada proporção de profissionais do ACeS PV/VC que menciona ter o rastreio oncológico atualizado de acordo com a sua idade
Comparando a proporção de cumprimento de cada um dos rastreios individualmente com a proporção média obtida para os utentes do ACeS nos meses de fevereiro a abril de 2015 (dados obtidos da plataforma

\begin{tabular}{|c|c|c|c|c|c|c|c|c|c|c|c|c|c|}
\hline \multirow{3}{*}{\multicolumn{2}{|c|}{ Características }} & \multicolumn{4}{|c|}{ Rastreio CCR } & \multicolumn{4}{|c|}{ Rastreio CCU } & \multicolumn{4}{|c|}{ Rastreio cancro da mama } \\
\hline & & \multicolumn{2}{|c|}{ Não } & \multicolumn{2}{|c|}{ Sim } & \multicolumn{2}{|c|}{ Não } & \multicolumn{2}{|c|}{ Sim } & \multicolumn{2}{|c|}{ Não } & \multicolumn{2}{|c|}{ Sim } \\
\hline & & $n$ & $\%$ & $n$ & $\%$ & $n$ & $\%$ & $n$ & $\%$ & $n$ & $\%$ & $n$ & $\%$ \\
\hline Género & $\begin{array}{l}\text { Homem } \\
\text { Mulher }\end{array}$ & $\begin{array}{r}3 \\
15\end{array}$ & $\begin{array}{l}25,0 \% \\
21,7 \%\end{array}$ & $\begin{array}{r}9 \\
54\end{array}$ & $\begin{array}{l}75,0 \% \\
78,3 \%\end{array}$ & $\begin{array}{r}0 \\
29\end{array}$ & $\begin{array}{r}0,0 \% \\
12,4 \%\end{array}$ & $\begin{array}{r}0 \\
205\end{array}$ & $\begin{array}{r}0,0 \% \\
87,6 \%\end{array}$ & $\begin{array}{r}0 \\
11\end{array}$ & $\begin{array}{r}0,0 \% \\
15,9 \%\end{array}$ & $\begin{array}{r}0 \\
58\end{array}$ & $\begin{array}{r}0,0 \% \\
84,1 \%\end{array}$ \\
\hline Estado civil & $\begin{array}{l}\text { Solteiro } \\
\text { Casado } \\
\text { Divorciado } \\
\text { Viúvo }\end{array}$ & $\begin{array}{r}3 \\
12 \\
3 \\
0\end{array}$ & $\begin{array}{r}30,0 \% \\
23,1 \% \\
15,8 \% \\
0,0 \%\end{array}$ & $\begin{array}{r}7 \\
40 \\
16 \\
0\end{array}$ & $\begin{array}{r}70,0 \% \\
76,9 \% \\
84,2 \% \\
0,0 \%\end{array}$ & $\begin{array}{r}8 \\
17 \\
4 \\
0\end{array}$ & $\begin{array}{r}14,3 \% \\
11,4 \% \\
14,3 \% \\
0,0 \%\end{array}$ & $\begin{array}{r}48 \\
132 \\
24 \\
1\end{array}$ & $\begin{array}{r}85,7 \% \\
88,6 \% \\
85,7 \% \\
100,0 \%\end{array}$ & $\begin{array}{l}1 \\
8 \\
2 \\
0\end{array}$ & $\begin{array}{r}11,1 \% \\
19,5 \% \\
10,5 \% \\
0,0 \%\end{array}$ & $\begin{array}{r}8 \\
33 \\
17 \\
0\end{array}$ & $\begin{array}{r}88,9 \% \\
80,5 \% \\
89,5 \% \\
0,0 \%\end{array}$ \\
\hline $\begin{array}{l}\text { Habilitações } \\
\text { académicas }\end{array}$ & $\begin{array}{l}\text { 1-4. }{ }^{\circ} \text { Ano } \\
5-9 .{ }^{\circ} \text { Ano } \\
10-12 .^{\circ} \text { Ano } \\
\text { Bacharelato/ } \\
\text { Licenciatura/ } \\
\text { Mestrado } \\
\text { Outras }\end{array}$ & $\begin{array}{l}1 \\
1 \\
6 \\
9\end{array}$ & $\begin{array}{r}50,0 \% \\
10,0 \% \\
40,0 \% \\
17,0 \% \\
\\
100,0 \%\end{array}$ & $\begin{array}{r}1 \\
9 \\
9 \\
44\end{array}$ & $\begin{array}{l}50,0 \% \\
90,0 \% \\
60,0 \% \\
83,0 \%\end{array}$ & $\begin{array}{r}0 \\
1 \\
4 \\
24\end{array}$ & $\begin{array}{r}0,0 \% \\
7,7 \% \\
10,5 \% \\
13,2 \%\end{array}$ & $\begin{array}{r}1 \\
12 \\
34 \\
158\end{array}$ & $\begin{array}{r}100,0 \% \\
92,3 \% \\
89,5 \% \\
86,8 \%\end{array}$ & $\begin{array}{l}0 \\
1 \\
3 \\
7\end{array}$ & $\begin{array}{r}0,0 \% \\
11,1 \% \\
21,4 \% \\
15,9 \%\end{array}$ & \begin{tabular}{r|}
2 \\
8 \\
11 \\
37
\end{tabular} & $\begin{array}{r}100,0 \% \\
88,9 \% \\
78,6 \% \\
84,1 \%\end{array}$ \\
\hline Função & $\begin{array}{l}\text { Enfermeiro } \\
\text { Médico } \\
\text { especialista } \\
\text { Médico interno } \\
\text { Assistente técnico } \\
\text { Técnico superior } \\
\text { de saúde } \\
\text { Assistente } \\
\text { operacional } \\
\text { Outra }\end{array}$ & $\begin{array}{l}0 \\
6 \\
0\end{array}$ & $\begin{array}{r}21,1 \% \\
17,1 \% \\
0,0 \% \\
31,6 \% \\
0,0 \% \\
25,0 \% \\
0,0 \%\end{array}$ & $\begin{array}{r}0 \\
13 \\
0\end{array}$ & $\begin{array}{r}78,9 \% \\
82,9 \% \\
0,0 \% \\
68,4 \% \\
0,0 \% \\
75,0 \% \\
0,0 \%\end{array}$ & $\begin{array}{l}4 \\
7 \\
1\end{array}$ & $\begin{array}{r}7,2 \% \\
23,4 \% \\
\\
11,1 \% \\
15,6 \% \\
11,1 \%\end{array}$ & $\begin{array}{r}32 \\
38 \\
8\end{array}$ & $\begin{array}{r}92,8 \% \\
76,6 \% \\
88,9 \% \\
84,4 \% \\
88,9 \% \\
100,0 \% \\
100,0 \%\end{array}$ & $\begin{array}{l}0 \\
4 \\
0\end{array}$ & $\begin{array}{r}10,5 \% \\
20,0 \% \\
\\
0,0 \% \\
22,2 \% \\
0,0 \%\end{array}$ & $\begin{array}{r}0 \\
14 \\
0\end{array}$ & $\begin{array}{r}89,5 \% \\
80,0 \% \\
\\
0,0 \% \\
77,8 \% \\
0,0 \%\end{array}$ \\
\hline
\end{tabular}




\begin{tabular}{|c|c|c|c|c|c|c|c|c|c|c|c|c|c|}
\hline \multirow{3}{*}{\multicolumn{2}{|c|}{ Características }} & \multicolumn{4}{|c|}{ Rastreio CCR } & \multicolumn{4}{|c|}{ Rastreio CCU } & \multicolumn{4}{|c|}{ Rastreio cancro da mama } \\
\hline & & \multicolumn{2}{|c|}{ Não } & \multicolumn{2}{|r|}{ Sim } & \multicolumn{2}{|c|}{ Não } & \multicolumn{2}{|c|}{ Sim } & \multicolumn{2}{|r|}{ Não } & \multicolumn{2}{|r|}{ Sim } \\
\hline & & $n$ & $\%$ & $n$ & $\%$ & $n$ & $\%$ & $n$ & $\%$ & $n$ & $\%$ & $n$ & $\%$ \\
\hline \multirow[t]{2}{*}{ Género } & Homem & 3 & $25,0 \%$ & 9 & $75,0 \%$ & 0 & $0,0 \%$ & 0 & $0,0 \%$ & 0 & $0,0 \%$ & 0 & $0,0 \%$ \\
\hline & Mulher & 15 & $21,7 \%$ & 54 & $78,3 \%$ & 29 & $12,4 \%$ & 205 & $87,6 \%$ & 11 & $15,9 \%$ & 58 & $84,1 \%$ \\
\hline \multirow[t]{6}{*}{ Onde exerce } & USF & 12 & $19,4 \%$ & 50 & $80,6 \%$ & 25 & $13,2 \%$ & 165 & $86,8 \%$ & 10 & $18,9 \%$ & 43 & $81,1 \%$ \\
\hline & URAP & 0 & $0,0 \%$ & 0 & $0,0 \%$ & 0 & $0,0 \%$ & 7 & $100,0 \%$ & 0 & $0,0 \%$ & 0 & $0,0 \%$ \\
\hline & UCC & 2 & $33,3 \%$ & 4 & $66,7 \%$ & 1 & $6,7 \%$ & 14 & $93,3 \%$ & 1 & $16,7 \%$ & 5 & $83,3 \%$ \\
\hline & USP & 3 & $60,0 \%$ & 2 & $40,0 \%$ & 2 & $25,0 \%$ & 6 & $75,0 \%$ & 0 & $0,0 \%$ & 3 & $100,0 \%$ \\
\hline & UAG & 1 & $16,7 \%$ & 5 & $83,3 \%$ & 1 & $8,3 \%$ & 11 & $91,7 \%$ & 0 & $0,0 \%$ & 5 & $100,0 \%$ \\
\hline & Outros & 0 & $0,0 \%$ & 2 & $100,0 \%$ & 0 & $0,0 \%$ & 2 & $100,0 \%$ & 0 & $0,0 \%$ & 2 & $100,0 \%$ \\
\hline \multirow{3}{*}{$\begin{array}{l}\text { Hábitos } \\
\text { tabágicos }\end{array}$} & Nunca fumou & 12 & $22,2 \%$ & 42 & $77,8 \%$ & 21 & $12,7 \%$ & 145 & $87,3 \%$ & 7 & $14,3 \%$ & 42 & $85,7 \%$ \\
\hline & Fumador & 5 & $35,7 \%$ & 9 & $64,3 \%$ & 5 & $12,2 \%$ & 36 & $87,8 \%$ & 4 & $33,3 \%$ & 8 & $66,7 \%$ \\
\hline & Ex-fumador & 1 & $7,7 \%$ & 12 & $92,3 \%$ & 3 & $11,1 \%$ & 24 & $88,9 \%$ & 0 & $0,0 \%$ & 8 & $100,0 \%$ \\
\hline \multirow[t]{2}{*}{ Hábitos etílicos } & Baixo risco & 18 & $22,5 \%$ & 62 & $77,5 \%$ & 29 & $12,4 \%$ & 205 & $87,6 \%$ & 10 & $14,7 \%$ & 58 & $85,3 \%$ \\
\hline & Consumo excessivo & 0 & $0,0 \%$ & 1 & $100,0 \%$ & 0 & $0,0 \%$ & 0 & $0,0 \%$ & 1 & $100,0 \%$ & 0 & $0,0 \%$ \\
\hline \multirow[t]{2}{*}{ Atividade física } & Sim & 0 & $0,0 \%$ & 1 & $100,0 \%$ & 0 & $0,0 \%$ & 0 & $0,0 \%$ & 1 & $100,0 \%$ & 0 & $0,0 \%$ \\
\hline & Não & 8 & $19,0 \%$ & 34 & $81,0 \%$ & 14 & $13,9 \%$ & 87 & $86,1 \%$ & 7 & $18,9 \%$ & 30 & $81,1 \%$ \\
\hline \multirow[t]{2}{*}{ Tem MF } & Sim & 18 & $22,2 \%$ & 63 & $77,8 \%$ & 28 & $12,3 \%$ & 199 & $87,7 \%$ & 11 & $15,9 \%$ & 58 & $84,1 \%$ \\
\hline & Não & 0 & $0,0 \%$ & 0 & $0,0 \%$ & 1 & $14,3 \%$ & 6 & $85,7 \%$ & 0 & $0,0 \%$ & 0 & $0,00 \%$ \\
\hline \multirow{3}{*}{$\begin{array}{l}\text { Consulta com } \\
\text { MF nos últimos } \\
\text { três anos }\end{array}$} & Sim & 16 & $20,8 \%$ & 61 & $79,2 \%$ & 21 & $10,5 \%$ & 179 & $89,5 \%$ & 10 & $14,9 \%$ & 57 & $85,1 \%$ \\
\hline & Não & 2 & $50,0 \%$ & 2 & $50,0 \%$ & 7 & $26,9 \%$ & 19 & $73,1 \%$ & 1 & $50,0 \%$ & 1 & $50,0 \%$ \\
\hline & Não se lembra & 0 & $0,0 \%$ & 0 & $0,0 \%$ & 0 & $0,0 \%$ & 1 & $100,0 \%$ & 0 & $0,0 \%$ & 0 & $0,0 \%$ \\
\hline \multirow{2}{*}{$\begin{array}{l}\text { Inscrito em } \\
\text { USF do ACeS }\end{array}$} & Sim & 18 & $23,1 \%$ & 60 & $76,9 \%$ & 20 & $11,7 \%$ & 151 & $88,3 \%$ & 11 & $16,7 \%$ & 55 & $83,3 \%$ \\
\hline & Não & 0 & $0,0 \%$ & 3 & $100,0 \%$ & 9 & $14,3 \%$ & 54 & $85,7 \%$ & 0 & $0,0 \%$ & 3 & $100,0 \%$ \\
\hline \multirow{3}{*}{$\begin{array}{l}\text { Medição de } \\
\text { tensão arterial } \\
\text { nos últimos } \\
\text { dois anos }\end{array}$} & Sim & 18 & $22,5 \%$ & 62 & $77,5 \%$ & 26 & $11,8 \%$ & 195 & $88,2 \%$ & 10 & $14,7 \%$ & 58 & $85,3 \%$ \\
\hline & Não & 0 & $0,0 \%$ & 0 & $0,0 \%$ & 0 & $0,0 \%$ & 7 & $100,0 \%$ & 0 & $0,0 \%$ & 0 & $0,0 \%$ \\
\hline & Não se lembra & 0 & $0,0 \%$ & 1 & $100,0 \%$ & 3 & $50,0 \%$ & 3 & $50,0 \%$ & 1 & $100,0 \%$ & 0 & $0,0 \%$ \\
\hline \multirow{3}{*}{$\begin{array}{l}\text { Vacina } \\
\text { antigripal no } \\
\text { ano anterior }\end{array}$} & Sim & 8 & $17,8 \%$ & 37 & $82,2 \%$ & 15 & $11,9 \%$ & 111 & $88,1 \%$ & 6 & $15,8 \%$ & 32 & $84,2 \%$ \\
\hline & Não & 10 & $28,6 \%$ & 25 & $71,4 \%$ & 14 & $13,2 \%$ & 92 & $86,8 \%$ & 5 & $16,7 \%$ & 25 & $83,3 \%$ \\
\hline & Não se lembra & 0 & $0,0 \%$ & 1 & $100,0 \%$ & 0 & $0,0 \%$ & 2 & $100,0 \%$ & 0 & $0,0 \%$ & 1 & $100,0 \%$ \\
\hline \multirow{3}{*}{$\begin{array}{l}\text { Vacina } \\
\text { antitetânica } \\
\text { atualizada }\end{array}$} & Sim & 18 & $22,2 \%$ & 63 & $77,8 \%$ & 28 & $12,2 \%$ & 201 & $87,8 \%$ & 11 & $15,9 \%$ & 58 & $84,1 \%$ \\
\hline & Não & 0 & $0,0 \%$ & 0 & $0,0 \%$ & 1 & $50,0 \%$ & 1 & $50,0 \%$ & 0 & $0,0 \%$ & 0 & $0,0 \%$ \\
\hline & Não se lembra & 0 & $0,0 \%$ & 0 & $0,0 \%$ & 0 & $0,0 \%$ & 3 & $100,0 \%$ & 0 & $0,0 \%$ & 0 & $0,0 \%$ \\
\hline
\end{tabular}

Legenda: ACeS - Agrupamento de Centros de Saúde; MF - Médico de Família; UAG - Unidade de Apoio à Gestão; UCSP - Unidade de Cuidados de Saúde Personalizados; UCC - Unidade de Cuidados na Comunidade; URAP - Unidade de Recursos Assistenciais Partilhados; USF - Unidade de Saúde Familiar; USP - Unidade de Saúde Pública.

SIARS® para a média dos respetivos indicadores flutuantes entre fevereiro e abril de 2015) verifica-se um cumprimento claramente maior pelos profissionais em todos os rastreios (CCU - 87,6\% vs. $66,2 \%$; cancro da mama $-84,1 \%$ vs. $68,2 \%$ e CCR - $77,8 \%$ vs. $65,3 \%$ ). No entanto, deve reter-se que a informação relativa aos 
profissionais foi autorreportada por oposição à obtida da plataforma que corresponderá a rastreios efetivos.

Quando se comparam os resultados obtidos com outros estudos efetuados com profissionais de saúde, em relação ao rastreio do CCU, o único estudo do género realizado no nosso país (PRECIT) ${ }^{12}$ teve lugar na Unidade Local de Saúde do Alto Minho e incluiu apenas médicas e enfermeiras (CSP e hospital) que reportaram uma adesão ao rastreio de $90 \%$, um número próximo do obtido no presente estudo ( $87,6 \%$ no global ou $87,3 \%$ se se considerarem só estas profissionais).

Internacionalmente, estudos nigerianos

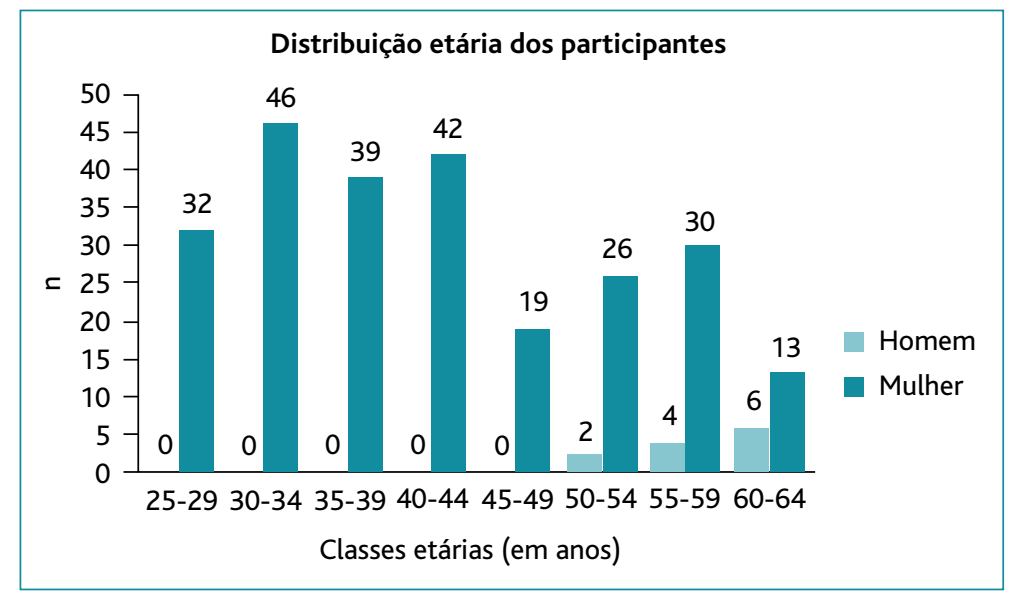

Figura 1. Distribuição dos participantes por idade e género $(n=259)$. que analisaram enfermeiras a trabalhar em meio hospitalar concluíram que $65,4 \%{ }^{13}$ a $79,5 \%{ }^{14}$ nunca tinham sido submetidas a colheita de citologia cérvico-vaginal. Já noutro estudo, decorrido na Coreia do Sul em médicas, enfermeiras e parteiras dos CSP, os resultados foram ainda mais baixos: apenas $13 \%$ tinha realizado citologia pelo menos uma vez, ${ }^{15}$ resultados próximos de um outro com enfermeiras hospitalares na Tanzânia, em que cerca de 15,3\% cumpriam o mesmo requisito; ${ }^{16}$ na Îndia, estudos chegaram a números entre 7 e 11,6\% nas mesmas condições. ${ }^{17-18}$ Estes dados contrastam com um artigo tailandês em que a taxa de cobertura da citologia cérvico-vaginal era de $79,5 \%$ na amostra testada, ${ }^{19}$ mais próxima dos resultados deste estudo e do PRECIT. ${ }^{12}$

Não estão disponíveis estudos portugueses relativamente aos restantes rastreios nestes profissionais. Assim, no que diz respeito a estudos internacionais e ao rastreio do cancro da mama, um estudo nigeriano com trabalhadoras de um hospital (médicas, enfermeiras, farmacêuticas, radiologistas e cientistas) mostrou uma taxa de cumprimento de apenas $3,1 \% ;{ }^{20}$ já outros estudos com funcionárias de hospitais verificaram que entre $3,6 \%^{21}$ e $80,3 \%{ }^{22}$ das mulheres analisadas tinha feito mamografia pelo menos uma vez. Por último, um estudo norte-americano verificou que apenas $46,6 \%$ dos médicos a exercer funções em CSP afirmavam cumprir o rastreio do CCR. ${ }^{23}$

Deste modo, os resultados extremamente variáveis tornam impossível comparar os resultados deste trabalho com os de outros, uma vez que tiveram lugar em

diferentes contextos (CSP $v$ s. hospitais), o leque de profissionais estudado diferiu e, a nível internacional, a realidade do rastreio oncológico e dos serviços de saúde também é distinta. $\mathrm{O}$ mesmo se pode dizer no que diz respeito aos motivos de não cumprimento e às relações encontradas no cruzamento do cumprimento dos rastreios com as características dos participantes, que não foram testadas noutros estudos pelo que não se podem estabelecer comparações.

No entanto, centrando mais a discussão nos médicos dos CSP, a adesão aos rastreios verificada contrasta com muitos resultados observados na literatura, em que apesar destes apresentarem uma atitude favorável em relação aos mesmos, acreditarem na sua importância e recomendá-los aos utentes, têm alguma reticência em cumpri-los. Dá-se, para isso, o exemplo de um estudo já referido em que a maioria dos médicos incluídos reconhecia a importância dos médicos, dos seus familiares subscreverem a realização de rastreios e dos médicos de família os recomendarem aos seus utentes, mas a maioria não cumpria o rastreio do CCR $(87,2 \%) .{ }^{9} \mathrm{As}-$ sim, apesar de não ter sido objetivo deste estudo, tendo em conta os resultados encontrados, os verificados nos utentes do mesmo ACeS e o facto de nenhum médico ter mencionado «Não acha importante» como motivo para não cumprimento de um rastreio, poder-se-á apoiar a possibilidade de existir uma consonância favorável entre o conhecimento, a atitude, a recomendação e o próprio cumprimento dos rastreios oncológicos pelos médicos. Estas conclusões não podem ser alarga- 
das aos outros grupos profissionais, não tão diretamente relacionados com a recomendação direta dos mesmos aos pacientes, apesar de o trabalho em equipa ser a base para a sua melhoria e de outros indicadores de desempenho com consequentes ganhos em saúde.

Identificaram-se algumas limitações neste estudo, entre elas, o facto de se ter usado um questionário não validado, que poderia induzir um erro de mensuração; para o minimizar foi realizado um estudo-piloto, dele resultando algumas alterações no questionário. Por outro lado, dependendo do autopreenchimento, poderá ter ocorrido um viés de memória, uma vez que várias questões reportavam a um período de tempo passado e um viés de desejabilidade social (apesar dos questionários serem anónimos) com os participantes a tenderem a responder o que é socialmente promovido/desejável, sobretudo tendo em conta que estes poderão estar mais informados acerca do que seria considerada a atitude correta para cada atividade ligada à prevenção da doença/promoção da saúde. Por último, poderá ter ocorrido um erro de má classificação, já que eventualmente se poderão ter considerado como não cumpridores participantes sem indicação para o rastreio; havia, no entanto, espaço para descrever essa hipótese nos motivos para o seu não cumprimento que pode, contudo, não ter sido devidamente utilizado por falta de literatura em saúde de alguns profissionais ou medo de exposição. Ainda assim, considera-se que tal não deverá ter influenciado significativamente os resultados, uma vez que se verificou um elevado cumprimento em todos os rastreios. Dando como exemplo o rastreio do CCU, ainda que se excluíssem as sete mulheres que assinalaram «Não tem colo do útero» ou «Outra razão», a percentagem de cumprimento seria de $90,3 \%$ em vez dos $87,6 \%$ encontrados.

Também não é possível excluir-se a existência de participantes que responderam mais que uma vez, o que parece pouco provável dado que a maioria foi preenchida no dia da entrega do questionário, logo após a sua distribuição por um dos investigadores (apenas sete foram colocados nas urnas).

Por outro lado, não foi possível analisar quais os fatores sociodemográficos e profissionais preditores para definição do perfil do profissional cumpridor (através de regressão logística multivariável), devido ao número reduzido de profissionais que afirmou não cumprir os rastreios. Anão existência “real” de dois grupos impossibilitou a comparação multivariável das características entre os que cumprem ( $n$ elevado) e os que não cumprem ( $n$ muito reduzido) o rastreio oncológico. No entanto, realce-se que este estudo não foi potenciado para esse objetivo.

Não se considera ter existido um viés de seleção dos participantes, uma vez que se tratou de um estudo censitário com uma elevada taxa de resposta, não se pretendendo extrapolar os resultados para os não respondedores. Poder-se-á levantar a questão acerca de quais destes participantes poderão ser considerados profissionais de saúde, sendo que mesmo o Portal da Saúde poderá gerar alguma ambiguidade na sua interpretação, mas parece apontar para que todas as carreiras incluídas sejam consideradas parte deste grupo. ${ }^{24}$

Deve-se ainda ressalvar o facto de ter ocorrido um alteração do bilhete de identidade dos indicadores em $2015,{ }^{25}$ com o acréscimo da alternativa Pelo menos um resultado de colpocitologia em meio líquido nos últimos cinco anos, que não parece que fosse ter um impacto significativo nos resultados, dados os valores encontrados.

Como pontos fortes deste trabalho destaca-se o facto de se tratar de um estudo censitário que incluiu uma população correspondente à de um ACeS com uma elevada taxa de resposta. Trata-se do primeiro estudo realizado em Portugal que avaliou o cumprimento dos três rastreios oncológicos preconizados pelo PNPCDO em profissionais de saúde e, nos casos em que era aplicável, os motivos para o seu não cumprimento. $\mathrm{O}$ trabalho debruçou-se exatamente sobre profissionais dos CSP. O que se torna particularmente interessante pelo facto de ser este o nível de cuidados responsável pela implementação de medidas preventivas da doença nos utentes e, deste modo, do rastreio oncológico. Assim, o estudo prima pelo facto de se focar na adesão dos profissionais a uma recomendação implementada ao nível de cuidados em que eles próprios desempenham funções. O desenho de estudo simples permite que este possa ser reproduzido noutros locais (já que este estudo não pretendeu ser inferencial para estes profissionais a nível nacional) e os resultados eventualmente comparados. A divulgação dos resultados poderá funcionar como um incentivo ao cumprimento por parte dos utentes.

\section{AGRADECIMENTOS}

À Dra. Julieta Gomes e aos elementos da USF Novo Cuidar, ACeS Alto Ave Guimarães/Vizela/Terras de Basto, que colaboraram no estudo-piloto. 
À Dra. Benedita Graça Moura pela colaboração no desenho do estudo e disponibilidade mostrada para o esclarecimento de dúvidas surgidas.

Aos intervenientes do Clinical Lab - Serviço de Consultoria para MGF prestado por Eurotrials, Consultores Científicos, em parceria com Merck Sharp \& Dohme e com patrocínio científico da Ordem dos Médicos.

\section{REFERÊNCIAS BIBLIOGRÁFICAS}

1. PORDATA. Óbitos por algumas causas de morte (\%) em Portugal [Internet]. Lisboa: PORDATA; 2016 [cited 2016 Jul; updated 2016 Apr 28]. Available from: http://www.pordata.pt/Portugal/\%C3\%93bitos +por+algumas+causas+de+morte+(percentagem)-758

2. Coordenação Nacional para as Doenças Oncológicas. Plano nacional de prevenção e controlo das doenças oncológicas 2007/2010 (PNPCDO): orientações programáticas [Internet]. Lisboa: Ministério da Saúde; 2007. Available from: http://www.iccp-portal.org/sites/default/files/plans/PNPCDO_2007_Versaofinal(1).pdf

3. Direção-Geral da Saúde. Programa nacional para as doenças oncológicas: orientações programáticas. Lisboa: DGS; 2012.

4. Granja M. Rastreio oncológico: conceitos, orientações e práticas [Cancer screening: rationale, guidelines and practices]. Acta Med Port. 2001;14(4): 441-7. Portuguese

5. Direção-Geral da Saúde. Rastreio oportunístico do cancro do cólon e reto: norma nº03/2014, de 31/03/14. Lisboa: DGS; 2014.

6. Ministério da Saúde e Administração Central do Sistema de Saúde. Bilhete de identidade dos indicadores de monitorização dos Cuidados de Saúde Primários no ano de 2014. Fevereiro 2014.

7. Wells $\mathrm{KB}$, Lewis $\mathrm{CE}$, Leake $\mathrm{B}$, Ware JE. Do physicians preach what they practice? A study of physicians' health habits and counseling practices. JAMA. 1984; 252(20):2846-8.

8. Pérula-de-Torres LA,Alonso-Arias S, Bauzà-Nikolai K, Estévez JC, IglesiasRodal M, Martín-Carrillo P, et al. Opiniones de los profissionales sanitários sobre la influencia del Programa de Actividades Preventivas y de Promoción de la Salud (PAPPS) en atención primaria [Opinions of health professionals on the impact of the Preventive Activities and Health Promotion Program (PAPPS)]. Aten Primaria. 2007;39 Suppl 3:5-14. Spanish

9. Peleg R, Ostermich A, Gienco V, Portughiez E. Screening tests among family doctors: do we do as we preach? Public Health. 2013;127(3):282-9.

10. Ribeiro C. Como atuar perante o consumo nocivo de álcool? Guia para cuidados de saúde primários [Internet]. Lisboa: Associação Portuguesa de Medicina Geral e Familiar; [s.d.]. Available from: http://www. apmgf.pt/ficheiros/GuiaCSP_ConsumoNocivoAlcool.pdf

11. Maiques-Galán A, Brotons-Cuixart C, Villar-Álvarez F, Martín-Rioboó E, Banegas-Banegas JR, Navarro-Pérez J, et al. Recomendaciones preventivas cardiovasculares. Aten Primaria. 2014;46 Suppl 4:3-15.

12. Regado B, Matos C, Barbosa C, Cadavez H, Faria I, Araújo S. Estudo PRECIT: prevalência de citologia cervical atualizada e fatores associados em enfermeiras e médicas da ULS Alto Minho [PRECIT Study: prevalence of current cervical cytology among nurses and physicians in the Alto Minho Local Health Unit]. Rev Port Med Geral Fam. 2014;30(6):360-6. Portuguese

13. Awodele O, Adeyomoye AA, Awodele DF, Kwashi V, Awodele IO, Dolapo DC. A study on cervical cancer screening amongst nurses in Lagos University Teaching Hospital, Lagos, Nigeria. J Canc Educ. 2011;26(3): 497-504.

14. Arulogun OS, Maxwell OO. Perception and utilization of cervical can- cer screening services among female nurses in University College Hospital, Ibadan, Nigeria. Pan Afr Med J. 2012;11:69.

15. Tran NT, Taylor R, Choe SI, Pyo HS, Kim OS, So HC. Knowledge, attitude and practice (KAP) concerning cervical cancer and screening among rural and urban female healthcare practitioners in the Democratic People's Republic of Korea. Asian Pac J Cancer Prev. 2011;12(11):3023-8.

16. Oranratanaphan S, Amatyakul P, Iramaneerat K, Srithipayawan S. Knowledge, attitudes and practices about the Pap smear among medical workers in Naresuan University Hospital. Asian Pac J Cancer Prev. 2010;11(6):1727-30.

17. Urasa M, Darj E. Knowledge of cervical cancer and screening practices of nurses at a regional hospital in Tanzania. Afr Health Sci. 2011;11(1):48-57.

18. Shekhar S, Sharma C, Thakur S, Raina N. Cervical cancer screening: knowledge, attitude and practices among nursing staff in a tertiary level teaching institution of rural India. Asian Pac J Cancer Prev. 2013; 14(6):3641-5.

19. Thippeveeranna C, Mohan SS, Singh LR, Singh NN. Knowledge, attitude and practice of the pap smear as a screening procedure among nurses in a tertiary hospital in north eastern India. Asian Pac J Cancer Prev. 2013;14(2):849-52.

20. Akhigbe AO, Omuemu VO. Knowledge, attitudes and practice of breast cancer screening among female health workers in a Nigerian urban city. BMC Cancer. 2009;9:203.

21. Nilaweera RI, Perera S, Paranagama N, Anushyanthan AS. Knowledge and practices on breast and cervical cancer screening methods among female health care workers: a Sri Lankan experience. Asian Pac J Cancer Prev. 2012;13(4):1193-6.

22. Abdullah NN, Aziz NA, Rampal S, Al-Sadat N. N. Mammography screening uptake among hospital personnel in Kuala Lumpur Tertiary Hospital. Asian Pac J Cancer Prev. 2011;12(10):2643-7.

23. Nodora JN, Martz WD, Ashbeck EL, Jacobs ET, Thompson PA, Martínez ME. Primary care physician compliance with colorectal screening guidelines. Cancer Causes Control. 2011;22(9):1277-87.

24. Serviço Nacional de Saúde. eProfissionalSaúde [homepage]. Lisboa: SNS; 2016. Available from: https://servicos.min-saude.pt/profissional/portal/

25. Administração Central do Sistema de Saúde. Bilhete de identidade dos indicadores de contratualização dos cuidados de saúde primários propostos para o ano de 2015 [Internet]. Lisboa: Ministério da Saúde; 2015. Available from: http://www.acss.min-saude.pt/Portals/0/bilhete_identidade_indicadores_contratualizacao_2015_2015_01_26.pdf

\section{CONFLITO DE INTERESSES}

Os autores declaram não ter conflitos de interesses.

\section{COMISSÃO DE ÉTICA}

Estudo realizado após parecer favorável da Comissão de Ética da ARS Norte.

\section{ENDEREÇO PARA CORRESPONDÊNCIA}

Margarida Moreira

Rua da Praia, n 186, Fieiro - Aguçadoura, 4495-031 Póvoa de Varzim

E-mail: mmargmoreira@gmail.com

Recebido em 05-11-2015

Aceite para publicação em 07-09-2016 


\section{ABSTRACT}

\section{COMPLIANCE WITH CANCER SCREENING AMONG PROFESSIONALS IN A GROUP OF HEALTH CENTRES}

Objectives: Cancer is the second leading cause of death in Portugal. The National Oncology Surveillance Plan includes screening programs for colorectal, breast and cervical cancer. The goal of this study was to assess compliance with cancer screening programs among professionals in a group of health centres.

Study design: Cross-sectional study.

Participants: All professionals in the ACeS Póvoa de Varzim/Vila do Conde group of health centres who were eligible for cancer screening.

Methods: Participants were asked to complete an anonymous questionnaire about their compliance with cancer screening programs or their reasons for non-compliance. The questionnaires were distributed between February and April 2015. Compliance was assessed based on the performance indicators for cancer screening used in primary health care in 2014.

Results: The response rate was $87.7 \%$ as 267 questionnaires were returned from 307 eligible participants. Eight questionnaires were excluded. The median age of participants was 41 years. Compliance with screening recommendations was found among $82.2 \%$. Compliance with colorectal cancer screening was found among $77.8 \%$, breast cancer screening in $84.1 \%$, and cervix cancer screening in $87.6 \%$.

Conclusions: Professionals in this population had good compliance with current cancer screening recommendations. This study may serve as a basis for comparison for future studies.

Keywords: Early Detection of Cancer; Health Personnel; Guideline Adherence.

\section{ANEXO I Questionário}

Caro Profissional do ACeS Póvoa de Varzim/Vila do Conde:

Somos um grupo de Médicos Internos e Especialistas de Medicina Geral e Familiar do ACeS Póvoa de Varzim/Vila do Conde que está a desenvolver um trabalho de investigação subordinado ao tema "Cumprimento do rastreio oncológico em profissionais de um ACeS".

Como tal, solicitamos a sua colaboração na resposta a este questionário que é destinado a ser preenchido por todos os profissionais do ACeS que se disponibilizem a preenchê-lo.

O preenchimento do questionário é anónimo e individual e todas as respostas são confidenciais. Estima-se que demore cinco minutos a completá-lo. Por favor, responda com sinceridade a todas as questões.

Após terminar agradecemos que o deposite dobrado na urna destinada a esse efeito.

1. Género:

Feminino

Masculino

2. Idade: (anos)

3. Estado civil:

Solteiro/a

Casado/a ou em união de facto
Separado/a ou Divorciado/a

Viúvo/a

4. Habilitações literárias:

Entre $1 .^{\circ}$ e $4 .^{\circ}$ ano do ensino básico

Entre $5 .^{\circ}$ e $9 .^{\circ}$ ano do ensino básico

$10 .^{\circ}-12 .^{\circ}$ ano do ensino secundário

Bacharelato/Licenciatura/Mestrado/Doutoramento

Outras 
5. Fuma pelo menos ocasionalmente um cigarro?

Sim

Não

6. É ex-fumador (de pelo menos um cigarro ocasional)?

Sim

Não

7. Relativamente às bebidas alcoólicas, por semana, em média, quantas unidades bebe de:

(escreva o número dentro do respetivo quadrado)

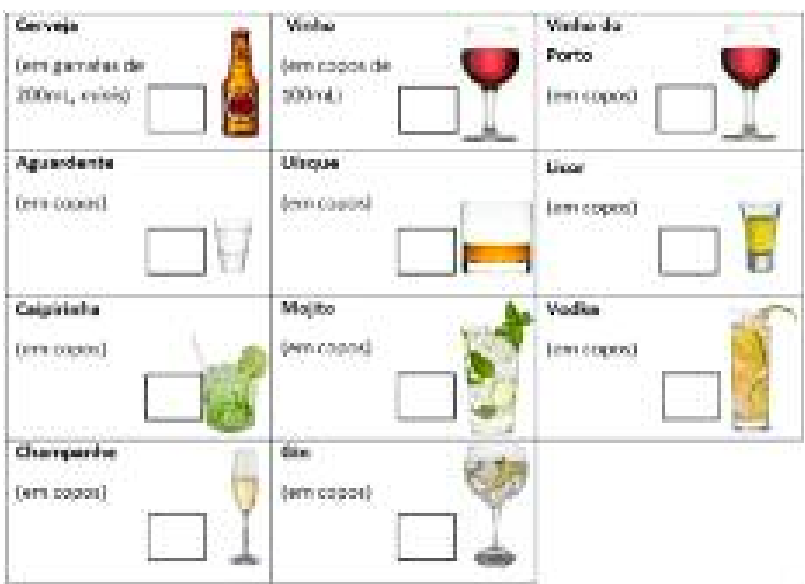

8. Pratica atividade física (caminhar, subir/descer escadas, jardinar, praticar desportos) em média mais de $\mathbf{3 0}$ minutos por dia, em pelo menos cinco dias da semana?

Sim

Não

9. Função exercida:

Enfermeiro/a

Médico/a especialista

Médico/a interno/a

Técnico superior

Assistente operacional

Assistente técnico

Outra

10. Onde exerce a sua atividade:

Unidade de Saúde Familiar (USF)

Unidade de Cuidados de Saúde Personalizados (UCSP)

Unidade de Recursos Assistenciais Partilhados (URAP)

Unidade de Cuidados na Comunidade (UCC)

Unidade de Saúde Pública (USP)

Unidade de Apoio à Gestão (UAG)

Outros
11. Está inscrito na lista de um médico/a de família?

Sim

Não

12. Está inscrito(a) numa USF do ACeS Póvoa de Varzim/Vila do Conde como utente?

Sim

Não

13. Teve pelo menos uma consulta presencial nos últimos três anos com o seu/sua médico/a de família?

Sim

Não

Não se lembra

Não aplicável

14. Foi-lhe medida a tensão arterial pelo menos uma vez nos últimos dois anos?

Sim

Não

Não se lembra

15. Foi-lhe administrada a vacina da gripe no ano passado?

Sim

Não

Não se lembra

16. Tem o Plano Nacional de Vacinação para o tétano atualizado?

Sim

$\square$ Não

Não se lembra

17. Se tem 50 ou mais anos, fez (assinale a resposta correta):

Pesquisa de sangue oculto nas fezes nos últimos dois anos

Colonoscopia total nos últimos 10 anos

Colonoscopia esquerda (retossigmoidoscopia) nos últimos cinco anos

Mais que um dos anteriores

Nenhum

17.1. Se respondeu "Nenhum", porque não fez? Não quis fazer

O seu médico não the recomendou

Falta de tempo

Esquecimento

Não acha importante. Porquê?

Outra razão. Qual? 
Se é homem ou se tem menos de 25 anos, o seu questionário acaba aqui. Muito obrigado pelas suas respostas.

Se é mulher com 25 ou mais anos, agradecemos que continue a responder.

18. Se tem entre 25 e 59 anos (inclusivé), fez pelo menos uma citologia cervico-vaginal ("papanicolau") nos últimos três anos?

Sim

Não

18.1. Se respondeu "Não", porque não fez? Não tem colo do útero

Não quis fazer

O seu médico não the recomendou

Falta de tempo

\section{Esquecimento}

Não acha importante. Porquê?

Outra razão. Qual?

19. Se tem entre 50 e 69 anos (inclusive), fez uma mamografia nos últimos dois anos?

Sim

Não

19.1. Se respondeu "Não", porque não fez?

$\square$ Não quis fazer

$\square$ O seu médico não the recomendou

$\square$ Falta de tempo

$\square$ Esquecimento

$\square$ Não acha importante. Porquê?

$\square$ Outra razão. Qual? 